\title{
IoT Based Power Line Communication in UndergroundCoal Mines
}

\author{
Ranjith.L.N,Lalnunsiama Fanai,KuengaChoeden,Sanjay Kumar.S,Paul Prassana Ku- \\ mar \\ Department Of Mining Engineering \\ Dr.T.Thimmaiah Institute Of Technology, KGF \\ pauledrttit.edu. in
}

\begin{abstract}
The intention of presenting this paper is to transmit/receive digital data via power lines for mining applications. Communications on the Power line use the existing power line network for connectivity purposes. This technology is preferred over other technologies. The advance power line communication network is planned to create "fast data rate and lower cost" connectivity in the remote region. Communication network which needs to cover the entire coal mine is difficult to establish. There's no useful technology currently available to solve this problem. As there are intrinsic benefits of the low-voltage power line in the underground coal mines, we plan to use it for communication. The sensed data of the gases underground is sent to the surface and is displayed in LCD then with help of IoT the result can be accessed throughout the world.
\end{abstract}

Keywords:Power line communication (PLC), sensed gases, internet of things (IoT), high data rate.

\section{Introduction}

Coal was formed some million years ago. Coal was one of the first heat and light sources for humans. The highest reserves are in the U.S., Russia, China and India. The Top 10 Coal Producers in The World are China $(3,874)$, United states of America (906.9), Australia (644.0), India (537.6), Indonesia (458.0), Russia (357.6), South Africa (260.5), Germany (185.8), Poland (137.1), Kazakhstan (108.7).The production of underground coal started with access tunnels, or adits, being mined from their surface outcrops into seams. [1][2] Apart from "air," which consists primarily78 percent of nitrogen and 21 percent of oxygen, there are four main gasses in a coal mine which concern workers. At underground coal mines, they are carbon dioxide (CO2), carbon monoxide (CO), methane (CH4), and hydrogen sulfide (H2S). In underground mines identification of gasses is necessary. Gas identification and communication network are complicated in underground coal mines. From the times of using humans, birds and safety lamps, we have changed to the usage of gas alarms, gas detection systems, gas sensors and continuous gas monitoring. [3] Common sensors include fuel gas sensors, detectors for photoionization, infrared point sensors, ultrasonic sensors, elec- 
trochemical gas sensors, and semiconductor sensors. [4] More recently, the use of infrared imaging sensors have increased. Most injuries are triggered by gases emitted in underground coal mines. Underground coal mines have a very hostile climate for the routine processes and events. Gas explosions remain the most severe type of incident in coal mine production [5].

The causes of these accidents were due to insufficient gas detection and inadequate communication systems. The need of the hour in underground mines both from safety and productivity point of view is communication. Proper and efficient communication networks not only save the equipment, they also help to move information quickly from the vicinity of the underground working area to the surface for rapid rescue. [6] Therefore, a reliable and effective communication system is also an essential requisite for safety in underground mines. We have also enhancedto wireless connectivity from the era of the use of existing pipelines and many types of telephones [7] [10]. The downside of using wireless communication is that, despite the substantial increase in the network's width or physical location, the investment required to construct it is greater due, among other factors, to the amount of cabling and the number of hardware ports that it needed. Wireless communication from underground mines to surface is theoretically proved but not practically. Of this reason, certain networks are uncommon. Since each system must be connected to each other and its installation and reconnection are difficult. We prefer Power Line Communication (PLC) to avoid this sort of problem. Connection with the power line is that we move the sensed data from underground to surface through existing power lines in the mine. Communications on power lines can be implemented at each time. Power line communication (PLC) technology is widely used over sinusoidal and continuous electrical networks and data rates up to several hundred megabits per second are guaranteed.Now-a-days, the power line is getting broad acceptance to give control and contact signals [8][9]. This has the benefit of requiring less initial costs to create a communication network. Power line demonstrated in this work can be used to transmit data using a simple communication interface with power line.

The findings say power line can also be used for data transmission at high speed. If high speed data transmission is possible overpower line, a microcontroller is the only system which can be programmed to generate a product according to applications. PLC networks differ considerably from the other conventional communication medium representing unfavorable environment for data transmission at high frequencies. Over the last years, a lot of research has been conducted investigating the indoor and outdoor PLC channel properties. Underground coal mining requires advance instrumentation for sustainable growth and safety of miners. Several measurements, such as gas emissions, strata states, temperature, air density, humidity, etc., need to be continuously tracked using sufficient sensors. Most coal mines in India are closed because of the risk of toxic gases. The sensors are connected to the PLC and warning input module, and the actuator is connected to the PLC output module. The gas sensor continuously measured concentration of gas present in underground mine atmosphere. 
The key reason for project selection is to create a new system that can be used effectively for tracking gasand other parameters in underground mines. Which is effective, advanced and cheap enough to be used in different mines, the main objective is to minimize accidents and other mine-related fire accidents that occur in mines due to negligence and unmonitored mine environment and also to establish a secure network of communication on the current power line.For our modem, we do not require additional cables or transmission wires, we have secure and wire-free communications to transfer the data to the surface. This brings some benefit and price decline that can be applied in underground mines.

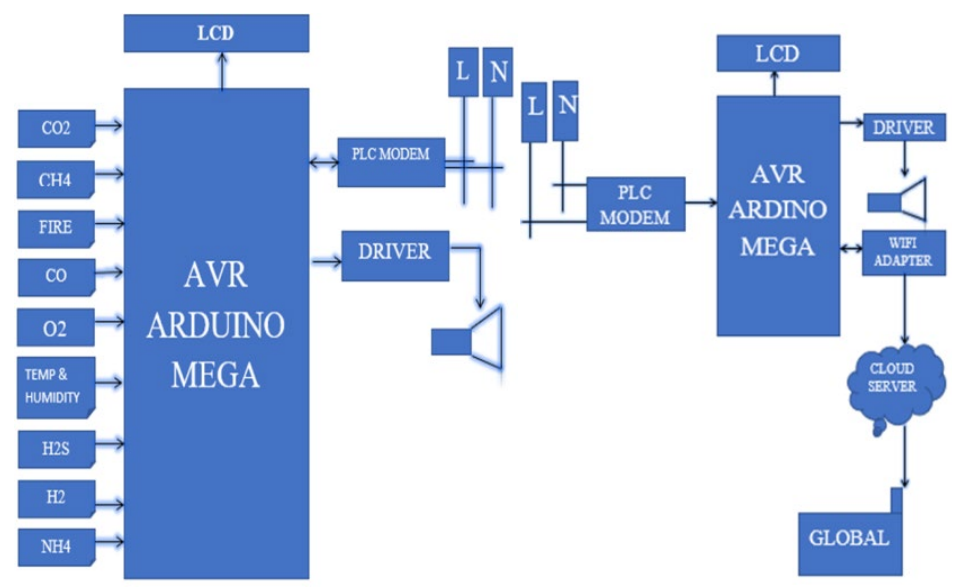

Fig. 1: Underground Block Diagram and Surface Block Diagram:

\section{$2 \quad$ Methodology}

The gases are sensed by the sensor, the analog data is converted by the microcontroller to digital data. Microcontrollers used are Arduino mega. Project design using Arduino mega gives you versatility to work with more memory space and processing power that allows you to work with a variety of sensors at once, and the data is sent to 3 microcontroller components. The data processed is sent to the LCD display and the driver first. The buzzer alarms beeps by audio if the gasses exceed their threshold value. Existing power lines which is present in underground mines is the source of media for transferring the data from underground to surface.Power line data transmission (PLDT) is the process of transmitting data over power lines: the three main steps are:

- Modulate the data so that the transmitting medium can be delivered. 
- Transmit the signal in such a way that the signal interference is reduced.

- The receiveddata is demodulated and the data is extracted.

A device capable of doing the above is called a power line modem. There are two PLC modems in use, one in the underground and the other on the surface. Using the PLC modem underground, digital data is translated into analog data and transmitted to the surface. On the surface the PLC modem transforms analog data to digital data again. The underground PLC modem acts as a transmitter and the surface PLC modem acts as a receiver.Medium voltage (MV), low voltage (LV) delivery network as the communication media for transmission of data, voices, real time image. Programming is done for the transmitting and receiving the data.

The programming is done in the Arduino software so with help of the program created data's can be transferred and received. The Arduino Integrated Development Environment (IDE) is a cross-platform framework, written in $\mathrm{C}$ and $\mathrm{C}++$ functions (for Windows ,MacOS, Linux). The data processed is even displayed on the surface LCD, and the buzzer alerts when the gasses cross their threshold value. Narrowband PLC runs at lower frequencies $(3-500 \mathrm{kHz}$ ), faster data speeds (up to $100 \mathrm{~s}$ per $\mathrm{kbps}$ ) and has a wider range (up to several kilometers), which can be expanded with repeaters. Broadband PLC runs at higher frequencies $(1.8-250 \mathrm{MHz})$, high data speeds (up to $100 \mathrm{~s}$ of Mbps), and is used in shorter-range applications. The data is transferred to the cloud via Wi-Fi adaptor. The data storage in cloud is known as "cloud storage".

This is based on internet of things (IoT). IoT is essentially a network of interconnected objects that are integrated with software sensors, network connectivity and the required electronics that allow them to capture and share data that makes them responsive. Many organizations have specific facilities for cloud-based data management and connectivity, for example, online services offered by Amazon. The server which we are using is Blynk Server. It acts as single way communication from server to user application. Here the server responds, and user takes the information from the server, as the server acts as a gateway for the flow of information. A mobile application called blynk is installed by students from Google play store.

A project dashboard is created. Arrange buttons, sliders,graphs, and other widgets are displayed on the screen. We can turn pins on /off using widgets, or display data from sensors. Although there are other applications to manage hardware over the internet, under an MIT license, blynk is one of the most accessible applications and also its free and open source. From the blynk cloud, the data can be viewed in the mobile phone. If the blynk application is installed in computer the continuous data received from the cloud can be viewed and monitored continuously. 


\section{$3 \quad$ Result}

Results obtained while implementing our project in the underground mine model of our college. In this proposed model the readings are taken at normal and abnormal environmental conditions by setting the threshold values at the above said program.

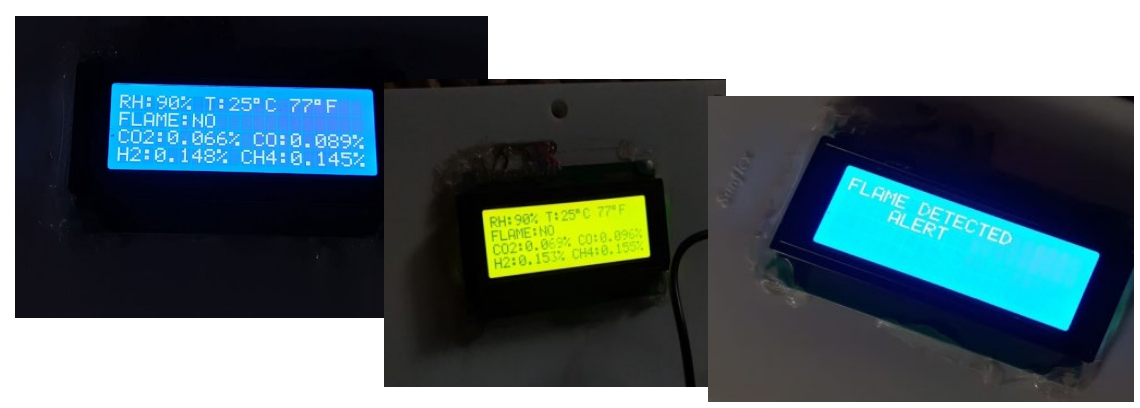

Fig 1(a): Readings shown on the undergroundLCD screen at normal environmental condition Fig 1(b): Readings shown on the surface LCD screen at normal environmental condition Fig 2(a): Alert shown on the underground LCD screen when flame is detected

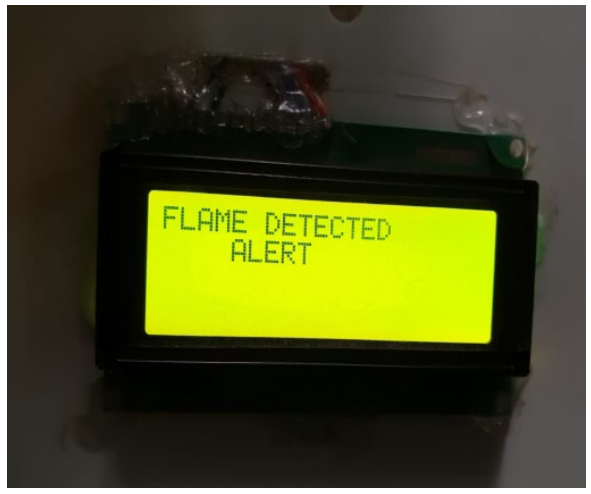

Fig 2(b): Alert shown on the surface LCD screen when flame is detected

The above figures 5.1(a) and figure 5.1(b) shows the readings displayed on LCD at normal environmental condition, and figure 5.2(a) and 5.2(b) shows the alert in underground and surface LCD screen. In the similar way, the readings are taken by using the sensors. These readings are noted by real time monitoring system and by using IoT, there will be delay of 1 second of IoT comparing to LCD. However these data's 
can be seen anywhere from the world. The main intension of our project is to monitor the gases and alert the underground miners working in there.

\section{Conclusion}

Throughout this report, we presented an initial step into an advanced early alert, incident monitoring and knowledge exchange network focused on IoT and power line communication that has the ability to provide robust security and enhanced services inside underground coal mine. To overcome the shortcomings of wireless communication, power line communication is proposed to be used. This new type of communication is very promising and will very efficiently be used to the future mining world.Sensor modules in Arduino have been selected to calculate eight parameters i.e. $\mathrm{CO} 2, \mathrm{CH} 4, \mathrm{CO}, \mathrm{O} 2, \mathrm{H} 2 \mathrm{~S}, \mathrm{H} 2, \mathrm{NH} 4$ and temperature and humidity at different underground mine locations with more than 95 percent and more than 99 percent accuracy and performance respectively.The findings from the introduction of the program specifically show the mine's status in terms of a "Mine Warning Scale" MWI 's contingency table is based on the cap value of gases, temperature, and humidity. Eventually, in creating IoT based power line communication for coal mines, this would be helpful in addressing problems of connectivity, serviceability, interoperability and versatility.

\section{References:}

1. Abdul Mannan, D.K. Saxena, MahrooshBanday, "AStudy on Power Line Communication” International Journal of Scientific and Research Publications, Volume 4, Issue 7, July 2014.

2. Aravindu.S, Athreya, Aqshay.C, Karthikeyan.J and Priya.B, "Design and Development of Coal Mine Safety System using IoT", International Journal of Recent Engineering Research and Development (IJRERD), Volume 02, Issue 07, July 2017, Pg. no. 97-101.

3. G. Prabhakar Reddy and M. Vijaya Lakshmi, "IOT in Mines for Safety and Efficient Monitoring", International Journal of Advanced Research in Computer Engineering \& Technology (IJARCET) Volume 4 Issue 11, November 2015.

4. KhaledMaaiufRabie, "Power Line Communication Systems", 2015, School of Electrical and Electronic Engineering Microwave and Communication Systems Research Group, page (10-180).

5. Pranav.M, Praveen. C, Monish. R, Prithvi Raj Mannur and Harsha. R "Power Line Communication of Sensor Data in Mines", International Journal of Latest Technology in Engineering, Management \& Applied Science, Volume VIII, Issue V, May 2019, ISSN 2278-2540.

6. S. S. Thouheed Ahmed, K. Thanuja, N. S. Guptha and S. Narasimha, "Telemedicine approach for remote patient monitoring system using smart phones with an economical hardware kit," 2016 International Conference on Computing Technologies and Intelligent Data Engineering (ICCTIDE'16), Kovilpatti, 2016, pp. 1-4, doi: 10.1109/ICCTIDE.2016.7725324.

7. Ahmed, S.T., Sankar, S. \& Sandhya, M. "Multi-objective optimal medical data informatics standardization and processing technique for telemedicine via machine learning ap- 
proach". J Ambient Intell Human Comput (2020). https://doi.org/10.1007/s12652-02002016-9

8. S. T. Ahmed and S. Sankar, "Investigative Protocol Design of Layer Optimized Image Compression in Telemedicine Environment", Procedia Computer Science, vol. 167, pp. 2617-2622, 2020, [online] Available: https://doi.org/10.1016/j.procs.2020.03.323

9. K. Vijayakumar, S. Suchitra and P. Swathi Shri, "A secured cloud storage auditing with empirical outsourcing of key updates", Int. J. Reasoning-based Intelligent Systems, Vol. 11, No. 2, 2019

10. K. Vijayakumar, Chokkalingam Arun, "Integrated cloud-based risk assessment model for continuous integration”, Int. J. Reasoning-based Intelligent Systems”, Vol. 10, Nos. 3/4, 2018. 